\title{
Selenium-vitamin E supplementation in infertile men: effects on semen parameters and pregnancy rate
}

This article was published in the following Dove Press journal:

International Journal of General Medicine

21 January 20II

Number of times this article has been viewed

\author{
Mohammad K \\ Moslemi ${ }^{1,2}$ \\ Samaneh Tavanbakhsh ${ }^{3}$ \\ 'Highly Specialized Jihad Daneshgahi \\ Infertility Center, Qom Branch \\ (ACECR), Qom, Iran; ${ }^{2}$ Department \\ of Urology, ${ }^{3}$ School of Medicine, \\ Qom University of Medical Sciences, \\ Qom, Iran
}

Objectives: Infertility is an important medical and social problem that has an impact on well-being. A significant development in the last 10 years in the study of human infertility has been the discovery that oxidative sperm DNA damage has a critical role in the etiology of poor semen quality and male infertility. Selenium $(\mathrm{Se})$ is an essential element for normal testicular development, spermatogenesis, and spermatozoa motility and function. The predominant biochemical action of Se in both humans and animals is to serve as an antioxidant via the Se-dependent enzyme glutathione peroxidase and thus protect cellular membranes and organelles from peroxidative damage. We explored the efficacy of Se in combination with vitamin $\mathrm{E}$ for improving semen parameters and pregnancy rates in infertile men.

Materials and methods: The study included 690 infertile men with idiopathic asthenoteratospermia who received supplemental daily Se $(200 \mu \mathrm{g})$ in combination with vitamin E (400 units) for at least 100 days. The mean age of cases was 28.5 years (range 20-45), and the median age was 30 years. These cases had presented with male factor infertility (primary or secondary) for at least 1 year. The longest and shortest duration of infertility was 10 years and 1 year, respectively. The median time of diagnosis of infertility was 1 year with a mean of 2.5 years.

Results: We observed 52.6\% (362 cases) total improvement in sperm motility, morphology, or both, and $10.8 \%$ (75 cases) spontaneous pregnancy in comparison with no treatment $(95 \%$ confidence interval: 3.08 to 5.52). No response to treatment occurred in 253 cases (36.6\%) after 14 weeks of combination therapy. Mean difference between semen analyses of cases before and after treatment was $4.3 \%$ with a standard deviation of 4.29 . On the basis of paired $t$-test results, combination therapy with oral Se and vitamin $\mathrm{E}$ was effective for treatment of asthenospermia or asthenoteratospermia or induction of spontaneous pregnancy $(P \leq 0.001)$.

Conclusions: Supplemental Se and vitamin E may improve semen quality and have beneficial and protective effects, especially on sperm motility. We advocate their use for the treatment of idiopathic male infertility diagnosed with asthenoteratospermia or asthenospermia in semen analysis.

Keywords: asthenospermia, sperm, semen, teratospermia, infertility, male, selenium, vitamin E

\section{Introduction}

The World Health Organization (WHO) defines infertility as the inability of a couple to achieve conception or bring a pregnancy to term after 1 year or more of regular, unprotected sexual intercourse. ${ }^{1}$ Conception is normally achieved within 12 months in $80 \%-85 \%$ of couples using no contraceptive measures. Although certain cases of male infertility are due to anatomical abnormalities, such as varicocele, ductal obstructions, or ejaculatory disorders, an estimated $40 \%-90 \%$ of cases are due to deficient sperm 
production of unidentifiable origin. ${ }^{2}$ Infertility is a major clinical concern, affecting $15 \%$ of all reproductive age couples. Male factors, including decreased semen quality, are responsible for $25 \%$ of these cases. ${ }^{3,4}$ Currently, the etiology of suboptimal semen quality is poorly understood, and many physiological, environmental, and genetic factors, including oxidative stress, have been implicated. ${ }^{5}$ Selenium (Se) is important for reproductive functions such as testosterone metabolism and is a constituent of sperm capsule selenoprotein. The administration of Se to subfertile patients induced a statistically significant rise in sperm motility. ${ }^{6}$ $\mathrm{Se}$ is an essential trace element occurring in organic and inorganic forms. The organic form is found predominantly in grains, fish, meat, poultry, eggs, and dairy products and enters the food chain via plant consumption. Typical dietary intake of Se in the US is $80-120 \mu \mathrm{g} /$ daily, and the recommended daily allowance is $70 \mu \mathrm{g}$ in men and $50 \mu \mathrm{g}$ in women. ${ }^{7}$ Selenoproteins participate in sperm structure integrity maintenance. Sperm capsular selenoprotein has an important structural role in spermatozoa in the form of glutathione peroxidase (GSH-Px). ${ }^{8}$ Increased reactive oxygen species (ROS) decreases fertility because ROS attacks the membrane of the spermatozoa, decreasing their viability. Increasing Se encourages antioxidant GSH-Px activity, thus decreasing ROS and leading to increased male fertility. ${ }^{9,10}$ Se could also protect against oxidative DNA damage in human sperm cells.

Vitamin $\mathrm{E}$ is one of the most important antioxidative molecules, residing mainly in the cell membranes. It is thought to interrupt reactions with lipid peroxidation and is a free radical scavenger generated during the univalent reduction of molecular oxygen and also normal activity of oxidative enzymes. ${ }^{11}$ These radicals will lead to peroxidation of phospholipids in the mitochondria of the sperm and thus to their ultimate immotility. ${ }^{12}$ It is possible that vitamin $\mathrm{E}$ enhances the production of the scavenger antioxidant enzymes. ${ }^{13}$

We studied the effect of daily oral supplementation of Se and vitamin $\mathrm{E}$ on the quality of semen parameters and pregnancy rate of couples with male factor infertility.

\section{Materials and methods Subjects}

From March 2007 to February 2010, 855 men with a mean age of 28.5 years (range 20-45 years) presenting with male factor infertility (primary or secondary) for at least 1 year were screened for enrollment in this prospective single-arm study in one center. Any infertile male with asthenoteratospermia according to WHO guidelines was enrolled in our study. Any patient with teratospermia of $1 \%$ or $2 \%$ in combination with $10 \%-30 \%$ asthenospermia was enrolled in the study. There was no known medical condition that could account for infertility. Only patients seeking medical attention for the disease were included in the study. The lowest age was 20 years, the highest age 45 years, and the median 30 years. The longest duration of infertility was 10 years; the shortest duration was 1 year. The median time of diagnosis of infertility was 1 year with a mean of 2.5 years. A total of 752 cases $(88 \%)$ had primary infertility, and 103 cases $(12 \%)$ suffered from secondary infertility (Table 1). Patients were included in the study after fulfilling certain criteria, including a sperm count of $>20 \times 10^{6} \%$ $\mathrm{mL}$, more than 1 year of failed attempt at conception, and no female factors. Exclusion criteria were abnormal testes on physical examination, such as bilateral or unilateral testicular atrophy; reproductive hormone levels (follicle stimulating hormone and luteinizing hormone) outside normal limits (increased or decreased in the case of primary testicular failure or secondary hypogonadotropic hypogonadism, respectively); genital diseases, such as undescended testes, varicocele; and history of genital surgery, oligo, or azoospermia. In the evaluation of cases, a history was taken, and on each patient we performed a physical examination. All patients who met study inclusion and exclusion criteria provided informed consent before study entry, which was done in accordance with the Declaration of Helsinki. ${ }^{14}$ The Human Ethics Committee approved the study protocol. Of the 985 screened patients, 855 cases met study inclusion and exclusion criteria and consented to proceed with the study protocol. The study consisted of a 14-week treatment phase. All patients were required to have ceased all medical therapy 6 weeks before study initiation. Eligible patients were assigned to fixed-dose treatment with $200 \mu \mathrm{g}$ oral Se tablet (L-selenomethionine) daily in combination with 400 IU synthetic vitamin E ( $\alpha$-tocopherol). Safety and tolerability of drugs were evaluated based on spontaneously reported adverse effects and physical examination

Table I Demographic data

\begin{tabular}{ll}
\hline Total number of cases & 855 \\
Lost to follow-up cases & 165 \\
Final number of eligible cases & 690 \\
Cases with primary infertility & 752 \\
Cases with secondary infertility & 103 \\
Mean age and range (years) & 28.5 and 20-45 \\
\hline
\end{tabular}


during each patient visit. Patients were asked to report all treatment-emergent adverse events. Treatment-emergent adverse events were defined as any adverse event that first occurred or worsened after the treatment trial or during it. Patients voluntarily reported adverse events throughout the study.

\section{Semen collections}

Semen was obtained from patients consulting the Highly Specialized Jihad Daneshgahi Infertility Center, Qom Branch (ACECR), which is affiliated to the Jihad Daneshgahi Center in Tehran, Iran. This center is the only infertility center in the province. After liquefaction of the sample, the semen volume, sperm concentration, and morphology of sperm were determined using standard procedures. ${ }^{15}$ In each case, we did a semen analysis before treatment and another semen analysis at the end of 100 days of treatment. Before semen sample collections, patients were instructed to abstain from ejaculation for at least $48 \mathrm{~h}$. Instructions regarding proper semen collection techniques were provided to patients at screening and throughout treatment. All procedures and interpretations were in accordance with 1992 WHO criteria, and morphology was established according to the Kruger parameters. Samples were assessed at least twice for volume, $\mathrm{pH}$, sperm concentration, progressive and total motility, and the percentage of normal forms. Two technicians were used. Standardized semen analysis techniques were taught to each technician at our infertility clinic. Normal WHO values included a sperm concentration of $20 \times 10^{6}$ spermatozoa $/ \mathrm{mL}$ or greater and $50 \%$ or greater motility with forward progression. Using Kruger strict criteria, males with $>14 \%$ normal forms were considered normal. Semen classification was as follows: normospermia was indicated by a sperm concentration of $\geq 20 \times 10^{6} / \mathrm{mL}$, motility $\geq 50 \%$, and $\geq 14 \%$ normal morphology; asthenospermia was indicated by a sperm concentration of $\geq 20 \times 10^{6} / \mathrm{mL},<50 \%$ motility, and normal morphology; and asthenoteratospermia was indicated by a sperm concentration of $\geq 20 \times 10 \% \mathrm{~mL},<50 \%$ motility, and $<14 \%$ normal morphology.

\section{Statistical analysis}

Statistical analysis was performed using SPSS software, Version 16 (SPSS Inc., Chicago, IL, USA). Values determined before and after treatment were compared by the paired $t$-test. For all comparisons, $P \leq 0.05$ was considered significant.

\section{Results}

The primary endpoint was the proportion of patients who had a $5 \%$ or greater increase in improvement of motility or morphology or both from baseline following the 14-week treatment period or impregnating their partner. A total of 165 patients withdrew from study prematurely after treatment initiation. The reasons for discontinuation were withdrawal of consent in 45 , missing data in 37 , and loss to follow-up in 83. All participants had abnormal sperm morphology using the Kruger strict criteria as well as asthenospermia. In 253 cases (36.6\%), no difference in semen analysis occurred. Normal pregnancy occurred in 75 cases $(10.8 \%)$. In 382 cases $(55 \%)$, improvement in parameters of semen analysis occurred. Improvement in motility of at least $5 \%$ occurred in 144 cases $(20.5 \%)$, motility improvement of more than $10 \%$ occurred in 155 cases $(22.5 \%)$, improvement in sperm morphology of at least $5 \%$ occurred in 21 cases $(3 \%)$, and improvement in both morphology and motility occurred in 42 patients $(6 \%)$ (Table 2). Mean difference between semen analyses of cases before treatment and after treatment was $4.3 \%$ with a standard deviation of 4.29 .

\section{Discussion}

Se is an essential trace element for humans and animals. ${ }^{16}$ Se is essential for sperm function and male fertility. Se deficiency has been linked to reproductive problems in rats, mice, chickens, pigs, sheep, and cattle, ${ }^{17}$ and supplementation with Se has been reported to improve reproductive performance in sheep and mice. ${ }^{18}$ However, high Se intake has been associated with impaired semen quality. ${ }^{19}$ This improvement is supplementation dependent, as all of the parameters returned to baseline values during the post-treatment period. ${ }^{20} \mathrm{Se}$ is incorporated into enzymes that regulate normal body processes. One Se-dependent enzyme is GSH-Px. GSH-Px protects cellular membranes and lipid-containing organelles from peroxidative damage by inhibition and destruction of endogenous peroxides, acting in conjunction with vitamin E to maintain integrity of these membranes. GSH-Px catalyzes the breakdown of hydrogen peroxide $\left(\mathrm{H}_{2} \mathrm{O}_{2}\right)$ and certain organic hydroperoxides produced by glutathione during the process of redox cycling. The toxicity of redox cycling compounds is generally increased by Se deficiency, which results in nearly a twofold increase in glutathione-Stransferase activity and glutathione synthesis in the liver. In order to protect human populations from excessive consumption of Se, $500 \mu \mathrm{g}$ of Se was generally accepted as 


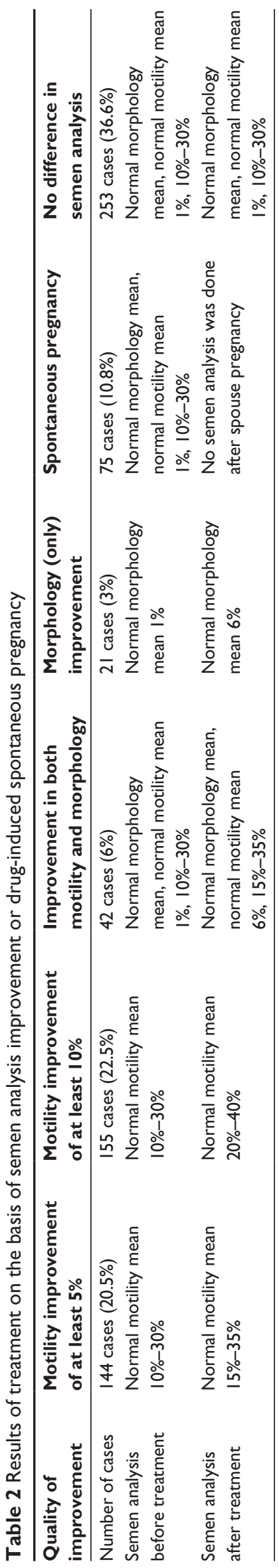

the maximum acceptable daily intake. Therefore, it can be predicted that chronic selenosis occurs with a daily intake of $\sim 1000-1500 \mu \mathrm{g}$ of elemental Se. ${ }^{21}$ Glutathione is vital to sperm antioxidant defenses and has demonstrated a positive effect on sperm motility. ${ }^{9}$ Se and glutathione are essential to the formation of phospholipid-hyperoxide GSH-Px, an enzyme present in spermatids that becomes a structural protein comprising over $50 \%$ of the mitochondrial capsule in the mid-piece of mature spermatozoa. Deficiencies of either substance can lead to instability of the mid-piece, resulting in defective motility. ${ }^{3}$

Vitamin E is a well-documented fat-soluble antioxidant and has been shown to inhibit free radical-induced damage to sensitive cell membranes. ${ }^{22}$ In one study, lipid peroxidation in the seminal plasma and spermatozoa was estimated by malondialdehyde (MDA) concentrations. Oral supplementation with vitamin E significantly decreased MDA concentration and improved sperm motility. ${ }^{13}$ The recommended dietary allowance of vitamin $\mathrm{E}$ is $15 \mathrm{mg}$ /day, and the tolerable upper intake level of any $\alpha$-tocopherol form is $1000 \mathrm{mg} /$ day. Although in most healthy adults short-term supplementation with up to $1600 \mathrm{IU}$ of vitamin E appears to be well tolerated and have minimal side effects, longterm safety is questionable. ${ }^{23}$ Vitamins $\mathrm{E}$ and $\mathrm{C}$ also play critical roles as nonenzymatic antioxidants. Vitamin E plays a vital role in protecting cell membranes from oxidative damage trapping and scavenging free radicals within cellular membranes. Vitamin $\mathrm{C}$ is a water-soluble antioxidant that reduces radicals from a variety of sources and also serves to recycle oxidized vitamin $\mathrm{E}$.

Suleiman et $\mathrm{al}^{13}$ investigated the effects of vitamin $\mathrm{E}$ on sperm motility. A total of 11 out of 52 treated patients (21\%) impregnated their spouses, and improved motility of sperm was seen in 31 subjects.

Oxidative stress is induced by ROS or free radicals. Although ROS have been shown to be required for sperm capacitation, hyperactivation, and sperm-oocyte fusion, ${ }^{21}$ excessive levels of ROS can negatively impact sperm quality. Increased levels of ROS have been correlated with decreased sperm motility, ${ }^{24}$ increased sperm DNA damage, ${ }^{25}$ sperm cellular membrane lipid peroxidation, ${ }^{26}$ and decreased efficacy of oocyte-sperm fusion. ${ }^{27}$ The pathological effects of oxidative stress arise under conditions in which levels of unscavenged ROS increase or the antioxidant buffering capacity of the system decreases, thus perturbing the delicate oxidantantioxidant balance. These free radicals induce sperm cell injury through several pathways and can significantly impact both sperm quality and function. ${ }^{27,28}$ 
In a systematic review of the effect of oral antioxidants (vitamins $\mathrm{C}$ and $\mathrm{E}$, zinc, Se, carnitine) on male infertility by Ross et $\mathrm{al}^{29} 17$ randomized trials, including a total of 1665 men, were identified. Of the 17 trials, 14(82\%) showed an improvement in either sperm quality or pregnancy rate after antioxidant therapy.

In one study, 69 infertile Scottish men were given placebo, Se, or Se in combination with vitamins A, C, and E for 3 months. At the end of the trial, both Se-treated groups had significant improvements in sperm motility; however, sperm density was unaffected. During the course of the study, $11 \%$ of the participants in the treatment groups impregnated their partner. ${ }^{30}$ This rate was similar in our study.

Hawkes et a $\mathrm{l}^{19}$ investigated the effects of dietary Se on sperm motility in 11 cases of healthy men. Interestingly, they found that high Se diet (297 $\mu \mathrm{g} /$ day) could impair sperm motility. However, our prescribed dose was $200 \mu \mathrm{g} /$ day, and our findings in a large series were the opposite.

\section{Conclusion}

After 14 weeks of treatment in 690 cases, mean motility increased significantly from baseline $(P \leq 0.005)$ compared with that before the treatment period. A slight but statistically significant increase in the normal morphological sperm ratio from baseline was observed at the end of the 14-week period.

Based on our findings, medical therapy of asthenoteratospermia with oral antioxidants, such as a combination of Se and vitamin E, can improve quality of semen parameters or help in achieving normal pregnancy. The major limitation of our study is the lack of a placebo-controlled, double-blind design. However, more studies with a case control design are warranted.

\section{Disclosure}

The authors report no conflicts of interest in this work.

\section{References}

1. Rowe PJ, Comhaire FH, Hargreave TB, Mahmoud AMA. WHO Manual for the Standardized Investigation, Diagnosis and Management of the Infertile Male. Cambridge (UK): Cambridge University Press; 2000.

2. Griffin JE, Wilson JD. Disorders of the testes. In: Isselbacher KJ, Braunwald E, Wilson JD, Martin JB, Fauci AS, Kasper DL, editors. Harrison's Principles of Internal Medicine. 13th ed. New York: McGraw Hill; 1994:2006-2017.

3. Ursini F, Heim S, Kiess M, et al. Dual function of the selenoprotein PHGPx during sperm maturation. Science. 1999;285(5432):1393-1396.

4. Sharlip ID, Jarow JP, Belker AM, et al. Best practice policies for male infertility. Fertil Steril. 2002;77(5):873-882.

5. Vine MF. Smoking and male reproduction: a review. Int J Androl. 1996; 19(6):323-337.
6. MacPhreson A, Scott R, Yates R. The effect of selenium supplementation in subfertile males. In: Anke M, Meissner D, Mills CF, editors. Proceedings of the Eighth International Symposium on Trace Elements in Man and Animals 1993. Jena (Germany): Verlag Media Turistik; 1993: 566-569.

7. National Academy of Sciences. Recommended Dietary Allowances. 10th ed. Washington (DC): National Academy Press; 1989:217-224.

8. Surai PF, Blesbois E, Grasseau I, et al. Fatty acid composition, glutathione peroxidase and superoxide dismutase activity and total antioxidant activity of avian semen. Comp Biochem Physiol B Biochem Mol Biol. 1998;120(3):527-533.

9. Irvine DS. Glutathione as a treatment for male infertility. Rev Reprod. 1996;1(1):6-12.

10. Klein EA. Selenium: epidemiology and basic science. J Urol. 2004; 171(2 Pt 2):S50-S53; discussion S53.

11. Palamanda JR, Kehrer JP. Involvement of vitamin E and protein thiols in the inhibition of microsomal lipid peroxidation by glutathione. Lipids. 1993;28(5):427-431.

12. De Lamirande E, Gagnon C. Reactive oxygen species and human spermatozoa. I. Effects on the motility of intact spermatozoa and on sperm axonemes. J Androl. 1992;13(5):368-378.

13. Suleiman SA, Ali ME, Zaki ZM, el-Malik EM, Nasr MA. Lipid peroxidation and human sperm motility: protective role of vitamin E. JAndrol. 1996;17(5):530-537.

14 Safarinejad MR, Safarinejad S. Efficacy of selenium and/or N-acetylcysteine for improving semen parameters in infertile men: a double-blind, placebo controlled, randomized study. J Urol. 2009;181(2):741-751.

15. World Health Organization. WHO Laboratory Manual for the Examination of Human Semen and Semen-Cervical Mucus Interactions. Singapore: Press Concern; 1980:7-32.

16. Hawkes WC, Turek PJ. Effects of dietary selenium on sperm motility in healthy men. J Androl. 2001;22(5):764-772.

17. Combs GF Jr, Combs SB. The Role of Selenium in Nutrition. San Diego (CA): Academic Press; 1986.

18. Tang CC, Chen HN, Rui HF. The effects of selenium on gestation, fertility, and offspring in mice. Biol Trace Elem Res. 1991;30(3): 227-231.

19. Hawkes WC, Alkan Z, Wong K. Selenium supplementation does not affect testicular selenium status or semen quality in North American men. J Androl. 2009;30(5):525-533.

20. Vézina D, Mauffette F, Roberts KD, Bleau G. Selenium-vitamin E supplementation in infertile men. Effects on semen parameters and micronutrient levels and distribution. Biol Trace Elem Res. 1996; 53(1-3):65-83.

21. Aitken RJ, Ryan AL, Baker MA, McLaughlin EA. Redox activity associated with the maturation and capacitation of mammalian spermatozoa. Free Radic Biol Med. 2004;36(8):994-1010.

22. Aitken RJ, Clarkson JS, Hargreave TB, Irvine DS, Wu FC. Analysis of the relationship between defective sperm function and the generation of reactive oxygen species in cases of oligozoospermia. J Androl. 1989; 10(3):214-220.

23. Vatassery GT, Bauer T, Dysken M. High doses of vitamin E in the treatment of disorders of the central nervous system in the aged. $\mathrm{Am} \mathrm{J}$ Clin Nutr. 1999;70(5):793-801.

24. Eskenazi B, Wyrobek AJ, Sloter E, et al. The association of age and semen quality in healthy men. Hum Reprod. 2003;18(2):447-454.

25. Armstrong JS, Rajasekaran M, Chamulitrat W, Gatti P, Hellstrom WJ, Sikka SC. Characterization of reactive oxygen species induced effects on human spermatozoa movement and energy metabolism. Free Radic Biol Med. 1999;26(7-8):869-880.

26. Aitken RJ, Harkiss D, Buckingham DW. Analysis of lipid peroxidation mechanisms in human spermatozoa. Mol Reprod Dev. 1993;35(3): 302-315.

27. Agrawal A, Prabhakaran SA, Sikka SC. Clinical relevance of oxidative stress in patients with male factor infertility: evidence-based analysis. AUA Update Ser. 2007;26:1-12.

28. Saleh RA, Agarwal A. Oxidative stress and male infertility: from research bench to clinical practice. J Androl. 2002;23(6):737-752. 
29. Ross C, Morriss A, Khairy M, et al. A systematic review of the effect of oral antioxidants on male infertility. Reprod Biomed Online. 2010; 20(6):711-723.
30. Scott R, MacPherson A, Yates RW, Hussain B, Dixon J. The effect of oral selenium supplementation on human sperm motility. $\mathrm{Br} J$ Urol. 1998;82(1):76-80.

\section{Publish your work in this journal}

The International Journal of General Medicine is an international, peer-reviewed open-access journal that focuses on general and internal medicine, pathogenesis, epidemiology, diagnosis, monitoring and treatment protocols. The journal is characterized by the rapid reporting of reviews, original research and clinical studies across all disease areas.
A key focus is the elucidation of disease processes and management protocols resulting in improved outcomes for the patient. The manuscript management system is completely online and includes a very quick and fair peer-review system. Visit http://www.dovepress.com/ testimonials.php to read real quotes from published authors. 